\title{
Causes and solutions to the psychological problems of the 90 s college students
}

\author{
Jianling Liu
}

Students Affair Department, Zhengzhou Institute of Technology

Address: No.18, Yingcai Street, Huiji District, Zhengzhou City, 450044, Henan Province, China.

Keywords: 90 s college students; psychological problems; psychological education; psychological health

\begin{abstract}
Nowadays, people who are active in the university campus are most 90s college students with distinct personality. 90s college students have a different side, but also face a lot of pressure. In recent years, more and more psychological problems of college students have appeared. This paper mainly describes the psychological problems and its solutions of 90s college students, hoping to give a reference to 90s college students, so that they can far away from psychological problems.

At the mention of 90s, words like "personalized publicity" and "strong self-consciousness" would come into our mind. However, 90s college students are also facing many problems. Based on learning pressures, social pressures and other factors, they are more likely to show more psychological problems. Therefore, in recent years, college student suicide, murder and other campus violence are increasing. The psychological problem of college students has become the focus of public attention, which has aroused the concern of the society. Extreme events caused by psychological problems of college students appear from time to time, which have brought great pain to countless families. Therefore, paying attention to college students' psychological problems, studying the causes of psychological problems of college students and taking necessary measures all have an important significance no matter for college students themselves, their family, school or society, which is also the current serious problem placed in front of the public.
\end{abstract}

\section{The main psychological problems existing in college students}

According to the relevant data shows that the psychological problems existing in 90s college students are mainly the following aspects:

1.1 The gap between ideal and reality

Before entering college, college students have had numerous fantasies and great longing for university life. But when they real enter into the university, they often feel frustrated because they find that there is a big difference between the actual university and their ideal university. Combined with a lot of inadaptation, it is easy to generate psychological problems like depression, irritability, etc.

\subsection{Personality and emotional problems}

A person's personality affects a person's ability to adapt to the new environment. A person who is not good at communication is less likely to integrate into a new environment than a cheerful one. And in new environment it will inevitably encounter all kinds of unsatisfactory things, which will enable them to produce negative emotions. If this negative emotions cannot be released in time, the more accumulated, the more difficult to extricate them, which will certainly bring a variety of psychological problems.

1.3 Interpersonal relationship problem

There are various problems existed in the interpersonal communication of college students: some students feel so good about themselves that they are dismissive to interact with others; some students feel inferior to others so that they fear of interaction with others; some students are withdrawn and not gregarious, so they are reluctant to communicate with others, etc. Thus it will causes strained interpersonal relationships between college students, and even they cannot properly communicate with people. 


\subsection{Academic pressure}

Compared with the study in senior high school, the study of the university is freer and more independent. After the completion of the course, the following a series problems such as the mastery of knowledge, thinking of the relevant problems, etc., are all completed by students themselves. University education lay more emphasis on the cultivation of autonomy and exploratory of college student learning. But some students cannot adapt this change temporarily, they feel very confused and overwhelmed, and finally leading to some psychological problems such as anxiety, schizophrenia, obsessive-compulsive disorder and so on.

1.5 Employment pressure

Nowadays, the employment of college students has become a social concern in society. With the sharp increase in the number of college graduates each year, for college students, although there are many self-employment opportunities, they still have to face with great employment pressure and bear more psychological burden. During the period of looking for a job, many college students cannot quickly find a suitable job, or even repeatedly rebuffed. Gradually, their employment passion would be consumed and then some psychological problems emerge.

\section{Causes of psychological problems of college students}

There are many reasons for the psychological problems of college students, and the main points are as follows:

2.1 Social environment

With the development of society, today's society has given great hope to college students and also put forward a very high demand. In order to adapt to social development and response to social demands, college students need to constantly improve their comprehensive quality, solidly master basic knowledge, practice hard personal knowledge and skills and fully improve themselves, so that they can be developed at all aspects. Therefore, they bear a huge psychological pressure. In fact, these pressures themselves will make college students feel depressed and distressed. In addition, with the increasingly complex social environment, network information has a great influence on college students, especially some negative information. It will seriously affect the physical and mental health of college students when they are unable to distinguish its danger.

\subsection{Family factor}

Since birth, 90s students have been regarded as the "honeypot generation". Their parents spoil them very much because most of them are the only child, resulting that most of the 90s are still in the psychological weaning period until the university stage. They may have developed a self-centered consciousness since childhood period, so they will feel out of favor once they are not be concerned, and then there will be anxiety, irritability and other psychological problems. Besides, influenced by the traditional concept of education, parents often expect their children to be success, which also gives them a great psychological pressure, and it is not conducive to the cultivation of health psychology. In the university stage, they still cannot independently face life, study and many other problems. Setbacks can easily lead to depression emotional reactions for them.

\subsection{School factor}

University is a place where students focus on learning knowledge. If the university's educational environment is too monotonous, the campus culture atmosphere is not enough, the school faculty is not enough, the teaching facilities are not complete and the gap between the university environment and the ideal is too large, it must will make students feel drab and boring, and then depression will arise spontaneously. However, when these psychological problems occur, if the school did not take effective counseling measures, more psychological problems will appear inevitably. 


\subsection{College students' themsel ves factor}

Some college students have bad personality characteristics, and their bad personality is the root cause of psychological problems. A person's personality characteristics can not only affect the way of thinking and behavior of this person, but determines the person's psychological ability. Therefore, a person's personality traits affect the mental health of the person. For example, the existence of extreme self-evaluation, and the inability to communicate normally. Of course, the factors that lead to their psychological problems may be genetic factors, or it may be caused by body lesions, etc. In short, if all kinds of bad characteristics of human nature could not be solved independently when college students in the face of setbacks, it will ultimately lead to the emergence of psychological problems.

\section{Measures to solve the psychological problems of college students}

In view of the psychological problems of present college students, we can solve it through the following aspects:

3.1 Purifying the social environment, and establishing a special psychological counseling institution for college students

With the development of modern society, the network information has a great influence on college students' psychology. Doing a good job in network management and purifying the network environment are conducive to creating a harmonious social environment. Great efforts should be made to combat the spread of numerous negative information through internet, so that to improve the cultural quality of the whole society. It is also necessary to learn the traditional spiritual culture, pay attention to social spiritual civilization, and optimize the social environment. Meanwhile, a special psychological counseling institution for college students should be established in order to do a good psychological counseling work of college students, establish and improve the university education system and do a good job in the supervision of college education.

3.2 Creating a harmonious family environment

Family is the most influential factor to the growth of children. Creating a harmonious family environment will help to cultivate the mental health of 90s college students. First of all, parents should lead by example. They should pay attention to their own cultural quality and moral character training, change their traditional concept, and focus on the development of children's own advantage education. Second, child's personality should be respect. Parents should adopt an equal dialogue with their children, to give them more spiritual support and care. Besides, a good living habit of children should be cultivated. The child's self-reliance should be cultivated consciously so that they can early get out of the weaning period and become a people with psychological maturity and behavior independent.

3.3 School: doing a good job in mental health knowledge education

In college education, college students' psychological education should be regarded as the main teaching content. Elective and compulsory courses for college students' psychological health education should be set up for college students. Mental health seminars should be held regularly, which can invite relevant experts to the scene to explain the knowledge of psychology and health education. What's more, television, network, school journal and other media can also be used to promote vigorously the mental health knowledge, strengthen the school culture construction, and create a good campus environment. For example, the organization of meaningful cultural and sports activities, carrying out the mental health day, psychological exchanges and other activities. With the universality of the network, it is possible to create a mental health website which belongs to college students themselves so as to play the important role of the network in the psychological education. Furthermore, psychological counseling phone can also be opened to listen to the inner thoughts of college students, and actively guide them out of the psychological haze. At the same time, a team of teachers with rich psychological knowledge can be established to help students solve the psychological problems caused by study, life, emotion, etc., making psychological health education as an important content of education in their teaching career. 
3.4 Strengthening and improving college students' psychological quality, and establishing a correct outlook on life values

College students should actively improve their own psychological quality. When faced with psychological problems, they should learn to self-regulate them actively. If they cannot adjust these psychological problems in time, they should take the initiative to find help, such as communicating with teachers and classmates, or counseling the professional psychological center. As long as they can release the negative emotions in time, find the right way to solve the problem, and meanwhile create their own cognition and correct behavior through the recognition and adjustment of negative emotions, they can regulate and solve by themselves even if they encounter the same problems in the future. In the meantime, college students should pay attention to the study of mental health knowledge in the weekdays, understand their own psychological characteristics, master the method of psychological adjustment, and consciously control their negative emotions.

All in all, society, school, family and personal factors all have important influence on college students' thoughts, psychology and behavior. Only by doing these four aspects, the development of college students' physical and mental health can be guaranteed. This paper has summarized the main psychological problems of college students, analyzed the causes of these psychological problems, and put forward some solutions to these problems, in the hope of having certain reference significance to improve the psychological quality of 90s college students.

Funding Project: Henan Province Soft Science Research Plan Projects "Local government performance evaluation system based on the balanced scorecard" (Project Number: 152400410312); Project supported by Science and Technology Innovation Team of Zhengzhou Institute of Technology.

\section{References}

[1] Wenjie Zhao. Mental Health of College Students [M]. Shanghai: Fudan University Press, 2004.

[2] Kai Hu. New View on College Students' Mental Health [M]. Changsha: Central South University Press, 2003.

[3] Guofu Tao, Xiangxing Wang. Social Psychology of College Students [M]. Sahnghai: East ChinaUniversity of Science and Technology Press, 2005.

[4] Chunling Xie. Analysis on the Social Causes and Countermeasures of the Psychological Problems of Contemporary College Students [J]. Education Forum. 2010(15):143-144

[5] Baozai Wang, Tieming Yu, Qinglong Zeng. Construction of "Trinity" of College Students' Psychological Health Education [J]. Modern Education Science. 2009(S1):300-301.

[6] Yueyun Wang, Hong Zhou, Ping Yin et al. Discussion on University Students' Psychological Health Problems and Its Countermeasures [J]. Chinese Journal of Social Medicine. 2007, 24(1): 29-30. 